\title{
Jeux, apprentissage - une bonne pratique avec des contes à l'école maternelle
}

\author{
Bereczkiné Záluszki, Anna \\ ELTE Tanitó- és Óvóképzö Kar Magyar Nyelvi és Irodalmi Tanszék
}

\begin{abstract}
Résumé
Les contes, outils quotidiens de l'enseignant, sont des ressources inépuisables en éducation littéraire. Cette étude offre quelques idées d'exploitation du conte populaire aux enseignants des écoles maternelles dans un contexte pédagogique pour encourager la communication libre et le plaisir de la création. Les bonnes pratiques donnent envie aux enfants d'écouter et de créer des histoires par épisodes et de communiquer avec les autres en apprenant la structure d'un conte populaire.
\end{abstract}

Mots-clés: littérature pour enfants, contes populaires, méthodologie, pédagogie des contes, apprentissage par jeux

Les contes traditionnels pendant des siècles ont enseigné les vertus sociales éprouvées et incontestées. De nos jours il existe un intérêt fort dans l'enseignement pour le folklore et surtout pour les contes, mais souvent un débat se crée parmi les chercheurs autour de l'actualité, de la modernité des contes. On entend des avis très différents, mais les psychologues et les éducateurs sont convaincus que les enfants ont besoin de contes authentiques, si on veut que leur vie psychique évolue d'une façon saine.

«Le passage à l'écrit des contes oraux opéra de la sorte un sauvetage paradoxal qui modifia irrémédiablement leur destination et en partie leur sens. Ce processus de déplacement et de distorsion, entamé en France par Charles Perrault, fit en effet basculer le conte dans la sphère enfantine.» (Bru, 2017) Il est le premier transcripteur qui, en France, s'est sérieusement appuyé sur la tradition orale, à la fin du XVII ${ }^{\mathrm{e}}$. Les Contes de ma mère l'Oye sont le recueil le plus répandu et le plus célèbre, ils sont riches de poésie, de symboles et fidèles dans leur structure, ainsi ils sont particulièrement propices à la lecture par épisodes en sauvegardant l'intérêt des enfants. On peut dire que c'est lorsque les contes de Perrault ont été écrits, à ce moment précis de l'histoire où de plus en plus d'auteurs européens commençaient à s'adresser explicitement aux enfants, donnant pour la première fois, par l'écrit, une forme 
concrète à des modèles, que s'amorça véritablement le développement de la littérature moderne pour enfants. Ce suspense ainsi créé permet de soutenir l'attention des enfants et de souligner en même temps les différentes fonctions du récit.

Il est essentiel d'évoquer l'importance des frères Grimm, car se sont eux qui ont rassemblé les contes populaires en Allemagne au début du XIXe siècle. Ils les ont repérés dans leur singularité et leur caractère propre d'œuvres intemporelles, anonymes, collectives, orales et par conséquent mouvantes, ils en ainsi ont pressenti la richesse. Ils y ont vu les restes de mythes anciens progressivement disparus et les traces de récits prestigieux dont la cohérence et l'intelligibilité se seraient peu à peu altérés dans le temps et l'espace de la transmission (Josiane Bru, 2017).

Ce genre est aujourd'hui une fiction littéraire qui aide les enfants à s'orienter dans la vie réelle tout en laissant ouvert le monde imaginaire. A travers les contes, grâce aux événements imaginaires présentés, les élèves peuvent sortir du monde quotidien, ce qui leur permet de maîtriser, dans la vie réelle, leurs propres émotions. Ce genre littéraire divertit, enseigne le bien, déclenche la peur ou, au contraire, l'apaisement, forme le caractère des élèves, et il occupe une place importante dans tout programme d'enseignement (Bereczkiné Záluszki, 2013).

Comme l'a souligné Bruno Bettelheim, les contes populaires, apparentés aux mythes anciens, ne sont en aucune manière remplaçables par des contes modernes. Dans son livre Psychanalyse des contes de fées, il nous montre que les contes répondent aux angoisses des enfants, en les informant des épreuves à venir et des efforts à accomplir. Pour que l'histoire accroche vraiment l'attention de l'enfant, il faut qu'elle le divertisse et qu'elle éveille sa curiosité. Mais pour enrichir sa vie, il faut en outre qu'elle stimule son imagination qu'elle l'aide à développer son intelligence et à voir clair dans ses émotions, qu'elle soit accordée à ses angoisses et à ses aspirations, qu'elle lui fasse prendre conscience de ses difficultés tout en lui suggérant des solutions aux problèmes qui le troublent (Bettelheim, 1976). Les contes offrent aux enfants une possibilité de mieux comprendre les obstacles, et les difficultés qu'ils affronteront dans la vie.

Les contes populaires sont des supports pédagogiques riches. Ils présentent de nombreux intérêts pédagogiques pour les enfants âgés de 3 à 7 ans, ils jouent un grand rôle dans la socialisation des enfants. Les contes favorisent de façon naturelle le développement de certaines qualités fondamentales, comme l'attention, la mémoire, l'expression, le vocabulaire et le jugement critique. Ils ont une valeur éducative au sens immédiat. L'enfant, par diverses activités autour des contes populaires développe ses capacités d'agir, de parler, de réfléchir et d'imaginer, élargit son expérience et augmente ses connaissances. Il répète à plaisir des mots dont les sonorités le charment et des comptines qui répondent à ses propres rythmes biologiques (Christophe \& Grosset-Bureau, 1994). 
Lâge préscolaire est caractérisé par la prédominance des émotions chez les enfants. Leur première rencontre avec la littérature se réalise principalement à travers des œuvres courtes et rythmées, leur intérêt pour l'algorithme dans le monde extérieur se renforce sans cesse, ils apprécient la pulsation des comptines et des chansons même lorsqu'ils ne peuvent pas comprendre leur sens (Bereczkiné Záluszki, 2013). En raison des caractéristiques d'âge, la joie de la rime et de la rythmique domine chez les tout petits enfants, andis qu'à l'âge de 5-7 ans il sont déjà plus intéressés par les contes de fées.

Aujourd'hui les contes populaires sont avant tout des textes, fixés par l'imprimé dans des livres pour enfants. Ils sont la forme la plus directe du récit, parce que les enfants sauront en repérer plus facilement la structure, les éléments communs de fabrication, mais aussi parce qu'ils les écoutent toujours avec beaucoup de plaisir. L'intérêt pour les contes populaires n'a cessé de grandir dans des établissements scolaires, les ateliers se sont multipliés dans ce domaine culturel particulièrement riche. Les pédagogues lisent aux enfants beaucoup de contes pour leur donner envie de raconter et de créer des nouveaux récits pleins d'expressions agréables, vivantes, riches, imaginatives et efficaces.

Les animations autour des contes peuvent fournir les cadres efficaces pour l'éveil aux autres langues, et aux autres cultures. Les enseignants sont intéressés aussi par l'influence du conte sur l'enfant, par sa valeur pédagogique, même dans l'apprentissage précoce des langues étrangères. Les observations de livres, et des objets dans les classes, font partie de l'apprentissage d'une langue. L'école maternelle met en présence de belles images, qui font rêver et engendrent d'autres images. Le travail avec les albums illustrés, les mimiques et les gestes, les postures de la maîtresse, démonstrations des objets dans la classe - tout aidera à la sensibilisation et à l'écoute des contes en langue étrangère, préparent à l'apprentissage de la compréhension d'un récit et de la communication structurée (Márkus \& Gölcz, 2018). Avec une éducation émotionnelle et artistique, nous pouvons obtenir d'excellents résultats, en utilisons la créativité des enfants. Il est préférable de raconter des histoires librement: c'est la meilleure façon de percevoir l'humeur du public, afin que la narration puisse être adaptée à la situation (Márkus, 2017).

Dans le cadre des activités scolaires habituelles, la création d'un conte permettrait d'assurer une synthèse des activités comme l'expression orale, le vocabulaire, la conjugaison, les exercices structuraux.

Tout au long de l'école maternelle, le maîtres et les maîtresses créent les conditions bienveillantes pour que tous les enfants (même ceux qui ne s'expriment pas ou peu) prennent la parole, participent à des situations langagières plus complexes que celles de la vie ordinaire.

La narration interactive à la maternelle peut être un pas en avant dans le domaine de la compréhension et de la création de récits, au cours de laquelle l'enfant apprend la relation entre le mot et la chose, l'enfant se comporte comme un « lecteur», avec une attitude participative et active (Szinger, 2009). Le jeu 
dramatique offre la possibilité de développer l'expression, de résoudre les problèmes, permet la découverte de $\mathbb{1}$ 冈autre et aide á la socialisation (Gönczöl, 2013). Jouer avec les textes de contes dans la classe, c'est raconter à nouveau une histoire entendue avec de nouveaux codes de communication.

Dans toutes les classifications des contes une distinction apparait entre les contes «merveilleux» et les autres. Ce type de contes populaires est très apprécié par les enfants dans la grande section de l'école maternelle. Ils les aiment par le fait qu'ils finissent bien : «ils se marièrent, vécurent heureux et eurent beaucoup d'enfants». Les obstacles sont surmontés, le méchant est puni. Les contes merveilleux, tout en donnant du plaisir à l'enfant l'aident à résoudre ses problèmes, l'éclairent et développent sa personnalité.

L'entraînement à la composition d'une histoire imaginée stimulé par des activités littéraires fait l'objet d'un travail méthodique en grande section. Vue d'une perspective didactique, le conte merveilleux présente de nombreux avantages: la relative simplicité de sa structure, et surtout sa valeur symbolique. L'étude la plus intéressante concernant la créativité et la liberté du conteur se trouve dans l'ouvrage de Vladimir Propp, la Morphologie du conte. En analysant les contes russes, Propp postule que tous les contes merveilleux ont une structure du même type. Il apporte une contribution considérable à l'analyse des contes. Il montre que toute tentative de classement est arbitraire si on ne tient pas compte de la structure. Il met en évidence le fait que derrière une représentation il y a des fonctions et des liens entre les personnages et des chaînes dramatiques. Or les fonctions correspondent à l'activité imaginaire des enfants et des adultes. Propp dit que la seule question importante est de savoir ce que font les personnages.

Enthousiasmés à juste titre par ces découvertes fondamentales -»le conte est un récit»-, les animateurs, et les enseignants pourront proposer des expériences créatives dans la classe s'appuyant sur un canevas issu des recherches de Vladimir Propp. Il porte l'accent sur la notion de fonction (l'action d'un personnage définie du point de vue de sa signification dans le déroulement de l'intrigue). Il définit 31 fonctions. Celles-ci ne sont pas toutes présentes dans un conte mais, selon lui, l'ordre dans lequel elles apparaissent est toujours le même. Les recherches pédagogiques nous ont conduits à nous concentrer sur quelques méthodes pratiques autour de l'utilisation des contes basés sur l'étude de Propp.

Nous essayons d'attirer l'attention sur quelques jeux, quelques possibilités d'utilisation des contes pendant les activités à la grande section de l'école maternelle, pour que les enfants aient le plaisir eux-mêmes de créer librement des histoires merveilleuses. Nous évoquons ici quelques pratiques pour le bon usage des contes. 


\section{La démarche pédagogique se construit en plusieurs étapes: écouter - analyser - créer}

I.

Les contes suscitent l'émotion, détendent, apportent l'émerveillement, le plaisir de la fiction. Il est important de raconter ou de lire beaucoup de contes merveilleux, particulièrement en grande section de l'école maternelle. Le conteur devra s'investir dans les descriptions, les invocations, les incantations, les formules, les dialogues, les éléments pratiques. Il est essentiel que la lecture des contes par le maître soit très attirante. Il est souhaitable qu'il récite des contes à haute voix, qu'il ne les interrompe pas, afin de maintenir l'attention des élèves tout au long de l'histoire. À travers la lecture le maître peut préparer les enfants de 6-7 ans à être dans une écoute active, en les aidant à développer leur capacité à écouter, à se projeter. Pour amener les élèves à développer ces compétences langagières, on les conduit à jouer avec les sons, la voix, les mots, les phrases. La connaissance du vocabulaire, les capacités de traitement de l'organisation textuelle sont essentielles dans la préparation pour la création d'un récit riche en expression.

II.

Les contes se déroulent entre un début et une fin clairement identifiable par l'auditeur en tant qu'éléments codés d'une stratégie narrative. Il expose un récit imaginé, les événements se situent dans une chronologie et s'articulent grâce aux mots de liaison relatifs: à l'espace, au temps, à la causalité, à la comparaison, à la discordance.

Avant de se lancer dans la création des histoires il est indispensable d'analyser la structure du conte merveilleux dans le cadre des animations interactives pour que les enfants appréhendent facilement la logique des éléments des contes de fées. Parallèlement avec l'imprégnation, un travail d'analyse du fonctionnement du schéma d'un conte fournira aux enfants les éléments et le cadre qui leur permettront de créer des histoires. A travers plusieurs exercices et jeux on fait apparaître la structure des contes merveilleux, ainsi on donne des éléments narratifs communs facilement assimilables pour être réinvestis. Il est très important que la maîtresse conduise un travail spécifique sur la compréhension qui s'élabore dans les échanges des idées et dans la communication autour du texte entendu.

Il est souhaitable que les enfants comprennent les étapes du récit, le passage d'une action du conte à une autre doit être compris. Pour faire comprendre la structure des contes, on peut organiser des démonstrations à l'aide de poupées marionnettes, en examinant ce qu'est un conte de fées, quelle est sa structure et quels sont ses composants. Ces activités ont pour but de développer la sensibilité, l'écoute et l'observation et de familiariser 
les enfants avec les formes artistiques les plus variées. Les élèves deviennent ainsi peu à peu sensibles aux règles qui régissent le conte et ils pourront exercer leur imagination en s'appuyant sur une structure préétablie. Ce travail d'imprégnation est essentiel avant la création de contes.

Il est très utile donc d'organiser une activité innovatrice pour préparer la création des contes. Il faut parler de l'enchaînement des éléments des contes merveilleux: sur le début de l'histoire, sur les lieux et les personnages, la succession des événements, sur la fin typique des aventures. Il est utile de montrer des formules magiques dans la conversation avec les enfants, de les inciter à jouer avec le temps grâce aux indicateurs temporels (il était une fois, il y a très longtemps, un jour ...). Il faut favoriser les questionnements des élèves sur le personnage principal, les liens avec d'autres personnages, leurs actions, leurs ressentis.

L'encouragement de la communication libre doit prendre une juste place dans l'ensemble des apprentissages prévus par le programme d'enseignement de l'école maternelle. Il est important de montrer l'attention que l'on porte à ce que les enfants disent, on doit solliciter l'avis des élèves, leur point de vue, leurs interprétations. Dans ces moments centrés sur la réception et la production orale, le maître devrait veiller à donner le temps nécessaire pour jouer avec le texte, stimuler la production de phrases complètes et structurées, et inviter l'élève à utiliser ses propres mots sur des sujet différents. Les enfants reprendront les contes lus et récités par le maître, inventeront des mots qui n'existent pas, joueront avec les mots usuels, les décomposeront, les recomposeront, - toutes ces expériences les aideront à inventer leurs propres histoires. Jouer avec les mots, avec des phrases développe la compréhension de messages et de textes entendus. Ce type d'enseignement produit rapidement des progrès dans la compréhension des contes traditionnels entendus.

\section{III.}

\section{Le conte comme un outil de jeu}

L'étape suivante du projet est de faire dessiner des jeux de cartes par les enfants. Cette démarche est proposée aux enfants après la phase de sensibilisation à la structure des contes déjà connus, entendus. Avec la mise en forme illustrée ils retirent quelques éléments constitutifs du conte merveilleux déjà connus et en même temps ils inventent de nouvelles idées dans leur travail créatif. Nous devrions leur préciser à l'avance qu'ils peuvent inventer librement des idées inspirées de la vie quotidienne et de n'importe quels éléments venus des contes écoutés, ou de leur fantaisie. Les enfants pourront bien se servir de leurs connaissances et de leur imagination pour bâtir plusieurs histoires en respectant la structure des contes de fées. Cette activité réalisée avec l'ensemble des enfants sert à favoriser la création d'histoires, et elle permet aux enfants d'établir la progression d'un récit et de se repérer dans la structure d'un conte. 
On propose en classe l'invention et la fabrication de cartes représentant un élément typique du conte de fée. Les activités requises pour mener ce projet se construisent à partir de quelques éléments constitutifs du schéma de Propp. Chaque élève réalise son propre jeu de cartes composé de huit pièces en dessinant les héros, les lieux, les obstacles, la fin de son histoire inventée etc. La création visuelle libre se compose de huit séances : les enfants créent une carte pendant chaque séance menée par le maître en suivant les étapes ci-dessous. Ils dessinent :

1. Un héros (par exemple: un prince, une princesse, un pêcheur, un enfant trouvé, etc.)

2. Le désir du héros, ou une chose qui lui manque pour être heureux (par exemple: un oiseau, une clé, une rencontre magique, un trésor, un animal magique, etc.)

3. Un conseiller-allié que le héros rencontre sur son chemin (par exemple: une fée, un magicien, un vieux sage, un voyageur, un savant, etc.)

4. Un objet magique qui permettra au héros de surmonter les épreuves qu'il subira (par exemple : un vieux document, une baguette magique, un livre, etc.)

5. Un obstacle que le héros rencontre et qu'il devra surmonter (par exemple: un ogre, une méchante reine, un dragon qui crache du feux, devinettes, duels, etc.)

6. Un ami (par exemple : un petit garçon, une princesse, un cheval) qui vient en aide au héros pour l'aider à réaliser son rêve, son désir quant il faut (par exemple: en le guérissant, en lui donnant un conseil, en le libérant d'un enchantement, en lui apprenant un secret etc.)

7. Un lieu, où se trouve ce qu'il cherche, le but final du voyage du héros, (par exemple : une forêt enchantée, des étoiles, le château, la mère, des champs, etc.).

8. La fin de l'histoire.

\section{Présentation des cartes}

Dans cette nouvelle étape, puisque chaque élève a déjà son propre jeu de cartes, on dispose de plusieurs collections d'images composées de dessins différents. Chaque élève présente ses cartes, donne son interprétation en expliquant son choix, les autres peuvent lui poser des questions. Toutes les interprétations sont acceptées. On peut choisir quotidiennement la collection d'images avec laquelle on peut jouer dans la classe et même dans la cour. Le but de cette activité est d'améliorer la capacité des enfants à s'exprimer, à créer une histoire en respectant l'ordre des fonctions et la construction d'un conte merveilleux avec un support visuel.

Les jeux qui libèrent l'imaginaire - variations dans le jeu de création d'histoires 
a/ Un enfant tire une image de chaque série des autres et ensuite invente individuellement une histoire avec les huit cartes pour la classe en suivant l'ordre du canevas d'un conte merveilleux.

b/Chaque élève choisit une carte, un participant commence l'histoire et, à tour de rôle les joueurs la complètent en ajoutant chacun son épisode. Le conte s'élaborera en laissant à chacun une possibilité de le prolonger à sa façon. Avec ces jeux de cartes créatifs fabriqués par les enfants on préserve le côté plaisir de la création d'un conte collectif, les enfants seront mis dans la même situation ludique. On donne aux enfants l'envie d'écouter des histoires créées par leurs camarades de groupe. Il est important de préserver le côté plaisir de construire des histoires par épisodes et de donner envie d'écouter la suite.

\section{Conclusion}

Il faut encourager la communication libre et le plaisir de la création dans toutes les activités dans les écoles maternelles. Les apprentissages sont favorisés par la richesse des relations qui se développent entre les enfants eux-mêmes, et entre les enfants et le maître. Les contes, outils familiers de l'enseignant, offrent des ressources privilégiées pour conduire l'enfant vers la découverte des livres, vers la littérature enfantine. Nous avons proposé ci-dessus quelques idées, parmi plusieurs autres possibles, d'exploitation du conte populaire dans un contexte pédagogique.

\section{Littérature}

Bereczkiné Záluszki, A. (2013a). Kortárs mondókák és versek az óvodai élményalapú irodalmi foglalkozásokon. In Podráczky, J. (Ed.), Müvészeti nevelés kora gyermekkorban: Módszertani kaleidoszkóp (pp. 37-50). Bethlen Gábor Alapkezelő Zrt.

Bereczkiné Záluszki, A. (2013). Une démarche pédagogique vers la compétence culturelle pour la sansibilisation á la lecture d'œuvres littéraires françaises. In. Árva, V. \& Márkus, É. (Eds.), Education and/und Forschung II. (pp. 363-370), ELTE Eötvös Kiadó.

Bettelheim, B. (1976). A mese büvölete és a bontakozó gyermeki lélek. Gondolat Könyvkiadó.

Christophe, S. \& Grosset-Bureau, C. (1994). Jeux poétiques \& langue écrite, Armand Colin.

Bru, J. (2017). Conte populaire français. Contes merveilleux. Supplément au Catalogue de Paul De-larue et Marie-Louise Tenèze. Établi par Josiane Bru, édité par Bénédicte Bonne-mason, postface de Nicole Belmont. Presses Universitaires du Midi.

Gönczöl, A. (2013). Ariadné fonala, a mesereprodukció formái. In Podráczky, J. (Ed.), Müvészeti nevelés kora gyermekkorban: Módszertani kaleidoszkóp (pp. 73-81). Bethlen Gábor Alapkezelő Zrt. 
Márkus, É. (2017). A mesepedagógia alkalmazási lehetőségei a kisgyermekkori idegennyelv-fejlesztésben (német jó gyakorlatok). In Márkus, É., M. Pintér, T. \& Trentinné Benkő, É. (Eds.), Jó gyakorlatok a korai idegen nyelvi fejlesztésben és pedagógusképzésben: Oktatás, kutatás, innováció (pp. 36-49), Eötvös Loránd Tudományegyetem Tanító- és Óvóképző Kar.

Márkus, É. \& Gölcz, M. (2018). A magyarországi német nemzetiség nyelvelsajátítási szokásai. Gyermeknevelés, 6(3), 56-69. https://doi.org/10.31074/201835669

Propp, V. (1995). A mese morfológiája. Osiris-Századvég Kiadó.

Szinger, V. (2009). Interaktív mesemondás és meseolvasás az óvodában a szövegértés fejlesztéséért. Anyanyelv-pedagógia 3., www.anyanyelv-pedagogia.hu/cikkek. php?id=184 


\section{Bereczkiné Záluszki, A.}

\section{Games, learning - a good practice with storytelling in kindergarten}

Stories, the teacher's daily tools, are inexhaustible resources for children in literary education. This study offers some ideas for using folk tales for preschool teachers in an educational context to encourage free communication and the enjoyment of creation. Good practices make children want to listen and create episode stories and communicate with others by learning the structure of a folk tale.

Keywords: children's literature, folk tales, methodology, storytelling pedagogy, game learning

Bereczkiné Záluszki Anna: https://orcid.org/0000-0003-0499-2599 